Partie, und der Schnitt verläuft nicht quer zu den Blutgefäßen, sondern in ihrer Längsrichtung; die Verletzung ist nicht, wie Schiferli glaubte größer, sondern geringer als beim Längsschnitt, ebenso die Blutung.

Wie immer glaubt mancher heute auf einem definitiven Plan angekommen zu sein; wie immer wird wohl auch hier die Zukunft noch manches Neue bringen oder Altes wieder zu Ehren ziehen.

$$
\text { R. v. Fellenberg. }
$$

\title{
Ein neuer Brief von Alfonso Corti (1822-1876)
}

Als im Jahre 1876 Alfonso Corti in Corvino San Quirico bei Casteggio starb, hat die wissenschaftliche Welt von seinem Tod keine Notiz genommen; kein Nachruf ist uns erhalten, kein Bericht über seine so erfolgreich begonnenen wissenschaftlichen Studien ist überliefert worden. Der Name Corti, den wir uns heute aus der Lehre vom Bau des Spiralorganes im inneren $\mathrm{Ohr}$ gar nicht mehr wegdenken können, war damals anscheinend nur noch Wenigen bekannt. Etwas mehr als 20 Jahre, während derer Corti sich im wissenschaftlichen Schrifttum nicht hatte vernehmen lassen, genügten schon, um die Erinnerung an den Mann auszulöschen, dessen 1851 erschienenes Werk «Recherches sur l'organe de l'ouie des mammifères» für die Anatomie wie für die Physiologie gleich wichtig gewesen ist.

In mühevoller Arbeit haben Brückner, Schaffer und Pincherle lange nach dem Tode von Alfonso Corti versucht, dessen Lebenslauf aufzuklären und insbesondere die Zeiten und Orte seiner wissenschaftlichen Studien zu ermitteln. Ihre durchaus fragmentarisch gebliebenen Untersuchungen habe ich erst vor kurzem in wesentlichen Punkten ergänzen können auf Grund von Briefen Cortis, die sich im Nachlaß des Berner Histologen und Physiologen G. G. Valentin (1810-1883) fanden. ${ }^{1}$ Soweit Cortis Lebenslauf für die medizin-historische Forschung bedeutsam ist, liegt er nun einigermaßen klar vor uns; gleichwohl verdienen neue Quellen noch unsere Beachtung, dies besonders dann, wenn sie auch über die Persönlichkeit und die Wesensart von Alfonso Corti weiteren Aufschluß bringen.

1 E. Hintzsche, Alfonso Corti. Berner Beiträge zur Geschichte der Medizin und der Naturwissenschaften Nr. 3, 1944. 
Das nachstehend mitgeteilte Schreiben Cortis gehört zu der früher von mir veröffentlichten Serie der Briefe an Prof. Valentin, den er von einem Studien-Aufenthalt in Bern her seinen Lehrer und Freund nennen durfte. Ich fand es erst kürzlich bei der weiteren Durchsicht der umfangreichen Briefsammlung Valentins, ${ }^{2}$ in der dieses Schreiben am falschen Ort eingereiht war und mir daher früher unter den vielen Hunderten von Blättern leider entgangen ist.

Zum Verständnis des Briefes mögen in Kürze einige Daten vorangestellt sein: Es ist immer aufgefallen, daß Corti nach seiner epochalen Arbeit über das Spiralorgan in der Schnecke der Wirbeltiere aus dem Jahre 1851 nur noch eine vom Ende des Jahres 1852 stammende kleine GelegenheitsUntersuchung publiziert hat. Tatsächlich konnte ich denn auch durch die neulich bekanntgemachten Briefe nachweisen, daß Corti vom Sommer $1851 \mathrm{ab}$ - nach dem Tode seines Vaters - den größten Teil seiner Zeit der Regelung von Familien-Angelegenheiten hat widmen müssen, und daß er zunächst dadurch der wissenschaftlichen Arbeit entfremdet worden ist. Allerlei Studienpläne beschäftigten ihn zwar auch damals noch, doch fand er nicht die Muße zu ihrer Ausführung. Erst im Herbst 1853 hat Corti erneut und mit Eifer seine anatomischen Untersuchungen wieder aufgenommen, nachdem Valentin ihm in Spätsommer dieses Jahres in Turin einen Besuch gemacht hatte. In der oben genannten Biographie von Corti deutete ich schon an, daß der zwölf Jahre ältere Valentin offenbar seinen ganzen Einfluß auf Corti dahin geltend gemacht hat, ihn wieder zu wissenschaftlicher Betätigung anzuregen.

Obwohl das nachfolgende Schreiben undatiert ist, kann doch mit Sicherheit angegeben werden, daß es aus der ersten Dezemberhälfte des Jahres 1853 stammt. Ich veröffentliche den zehn Oktav-Seiten langen Brief in der Schreibweise des Originales und ungekürzt, da auch die einleitend erwähnten Erörterungen über eine eventuelle Berufung von Hipp nach Turin von Interesse sind. Matthäus Hipp (1813-1893) war ein zu seiner Zeit berühmter Mechaniker und Apparate-Bauer; er war 1852 vom Schweizerischen Bundesrat zum Vorsteher der Telegraphen-Werkstätte gewählt worden; später war er Leiter einer eigenen Telegraphenfabrik in Neuenburg. Von seinem Ansehen in naturwissenschaftlich interessierten Kreisen zeugt z. B. die Tatsache, daß die Mitglieder der Schweizerischen Naturforschenden Gesellschaft gelegentlich ihrer Tagung im

2 Stadt- und Hochschulbibliothek Bern, Mss. Hist. Helv. XXVIII 64-66. 
Jahre 1866 seiner Werkstätte in Neuenburg einen Besuch abgestattet haben (M. Perty, Erinnerungen, 1879, S. 222). Aus dieser Mitteilung ist schon zu ersehen, daß die in dem Briefe erörterte Berufung von Hipp nach Turin nicht zustande gekommen ist. Ich will jedoch nun dem Inhalt des Briefes nicht weiter vorgreifen und lasse Corti selbst zu Worte kommen; er schreibt:

[Ohne Ort und Datum, anscheinend in der ersten Dezemberhälfte 1853 aus Turin geschrieben.]

Carissimo e pregiatissimo Amico,

Scuserà se varie occupazioni contenziose mi costrinsero a ritardare fino ad oggi la mia risposta. Primieramente risponderò in Tedesco come potrò all'affare Hipp-Torino.

1. Wie viel kann er brauchen um mit Familie in Turin zu leben? Ich habe mich bei Damen erkundigt, und es wurde mir geantwortet, $4-5$ tausend Franken jährlich. Denn in Turin es ist bedeutend theurer als in Bern. Aber auch in dieser Beziehung sollte sich Mr. Hipp selbst in Turin umsehen. Ich würde ibm auch behülflich sein. Denn - wie weiter unten - muß er zuvor auf jeden Fall allein nach Turin kommen.

2. Kann er auf Bonelli's Wort ohne weiteres nach Turin kommen? Das werde ich nie rathen. Ich bin mit Bonelli bekannt wie mit Hunderten Anderen. So viel ich weiß kann ich Bonelli nur loben. Aber - unter uns gesagt - auf dieser Welt an Niemanden trauen!' So spreche ich mit einem Freunde. Wie soll er allso thun? Er muß hieher kommen und nachdem er mit Bonelli einverstanden ist — sich wechselseitig mit einem förmlichen Vertrag (istrumento) binden, der vor den Gesetzen volle Gültigkeit habe.

Jetzt noch ein Paar confidenzielle Bemerkungen.

1. Wenn H. vom Minister so angestellt werden könnte wie er sagt, so meine ich, es wäre ein gutes Ding, und würde H. - wenn er klug und pfiffig genug umzugehen versteht — auch ein ordentliches Honorar beziehen können. Er darf aber nicht vergessen, daß dieser Minister l'homme plus consumé dans la ruse ist, den ich kenne, ferner daß bei uns die Ministern sehr oft gewechselt werden. Auch deßwegen sollte allso H. a) sich die Reise-Kosten zahlen lassen, b) nach Turin allein kommen und - wenn er auch mit dem Minister einverstanden ist - sich ein förmliches vom König unterzeichnetes Amts-Diplom geben lassen.

3 Wohl: kann man Niemandem trauen. 
Nach allen diesen nothwendigen Formalitäten werde ich ihm anrathen, wenn es ihm conveniert - hieher zu übersiedeln. Ich spreche aus Erfahrung, denn ich habe schon eine ähnliche Angelegenheit geführt, um den Optiker Henkel in Bonn her zu rufen. Diese Herren haben immer sehr schöne Worte: die Thatsachen aber sind problematisch.

2. Die Übersiedlung nach Turin könnte aber für Hipp auch folgende gute Seite haben, die De-Filippi sehr hoch anschlägt. Wir haben in OberItalien keinen Mechaniker, der nicht ein $\mathrm{Vieh}^{4}$ ist, und das Verlangen nach Instrumenten ist sehr groß von Seite mehrerer Professoren der Physik und der Astronomie, die jetzt in dieser Beziehung verzweifelt sind. De-Filippi meint allso, daß Mr. Hipp in Turin nicht einmal alle Bestellungen annehmen könnte. Was mich anbelangt - wenn nicht so viel doch bedeutende Bestellungen glaube ich auch, daß er erhalten würde. Beim Baron Plana, einem intimen Freunde von mir (Prof. d. Astronomie etc. etc.) würde ich ihn sehr empfehlen. So thäten wir auch bei den Professoren von Pavia, Mailand, Genua, Pisa u. Florenz. Zur Conclusion. Mein freundschaftlicher Rath ist folgender: Mr. Hipp soll sich brieflich beim Minister melden um sich die Reise-Kosten decken zu lassen, dann her kommen, und beide Stellen annehmen, wenn sie mit einem mäßigen Gehalte verbunden sind. Dann erst definitiv her übersiedeln, wobei er auch die von De-Filippi erwähnten möglichen Bestellungen wohl berücksichtigen soll. Endlich stelle ich mich Ihnen und Mr. Hipp zur Disposition in Allem, wo ich behülflich sein könnte.

La ringrazio infinitamente per la gentilezza colla quale ha voluto propormi una Machinerie per adoperare l'elettricità sotto il microscopio non che per l'offerta di farmela eseguire. Per ora però pensai de m'en passer convenendo con Lei che sarebbe un Luxusartikel. In ogni caso se Mr. Hipp viene a Torino potrò sempre farmela eseguire quà secondo il di Lei disegno. E mille grazie nuovamente per la di Lei estrema compiacenza.

Ella mi scrive che sta schwitzend über Ihren Jahresbericht. Non intendo di quale voglia parlare. Perocchè io possiedo già il Canstatt's Jahresbericht über die physiologischen Wissenschaften in J. 1852 ove si trova anche il suo Bericht über die Physiologie. Si sta forse già preparando un nuovo Jahresbericht pel 1853? Sarei molto curioso di saperlo.

Mentre Ella cortesemente mi propone alcuni quesiti intorno alle torpedini,

4 Vulgäre Ausdrucksform als Folge der unzureichenden Beherrschung der deutschen Sprache, gemeint ist etwa: Dummkopf. 
mi cita un nuovo lavoro di Remak sulle sue organische Fasern nelle quali avrebbe trovato l'Axenzylinder. La pregherei di dirmi alla prima occasione dove si trova questo lavoro che grandemente mi interessa pel seguente motivo. In questi giorni ricevetti una torpedo da Genova. Studiai la terminazione dei nervi nell'organo elettrico e non vidi finora che freie Endigungen. Keine einzige End-Anastomose come credevo di aver veduto prima. Mi feci poi a ricercare il ganglion splanchnicum mentovato da R. Wagner, ed il simpatico. Crederebbe? Non mi fu possibile trovare il simpatico degli autori, vale a dire un funiculus albicans ad latera columnae vertebralis decurrens la di cui struttura microscopica fosse veramente nervosa. Trovai in vece precisamente nella regione dove decorrerebbe il simpatico un cordoncino i di cui caratteri macroscopici concordavano perfettamente con quelli del simpatico degli autori, id est color, volumen, situs, decursus et ramificatio? Al microscopio non ricettava che del purissimo Sehnen-Gewebe! Nelle parte superiore si ramificava sul mesogastrio, o nella inferiore vidi palesemente che dipartiva da un grosso tendine (in ciascun lato) il quale serviva di inserzione a varii muscoli del bacino (Becken) e della coda. L'ufficio di questo tendine sarebbe adunque di ritenere in sito il peritoneo ed i visceri del basso ventre. Fra i vasi dell'addome e del torace nessuna traccia di nervo simpatico. Nella regione del collo trovai solo un anastomosi retiforme fra le radici del primo nervo spinale e la radice del vagus e credo anche del trigeminus. Non ostante un esame diligente, ma sopra un solo esemplare credo ancora den Sympaticus übersehen zu haben. Però la presenza di quel funiculus tendineus e la descrizione problematica che da Leydig (Über die Rochen und Haie) della struttura microscopica del sympaticus delle torpedini rendono la cosa interessante. Tanto più che io non posso ancora persuadermi della natura nervosa delle Remakschen Fasern. Anche la descrizione che da Koelliker di quelle fibre e del simpatico in genere è un ibis et redibis non.

Questa circostanza mi persuase ad intraprendere un esame accurato delle Remaksche Fasern e del simpatico in genere. Imperocchè bisogna confessare che al giorno d'oggi siamo ancora affatto all'oscuro intorno la vera struttura del simpatico. Conto di intraprenderne la ricerca in varii animali e finire poi colla monografia di un simpatico di un piccolo animale nel quale potrò esaminare tutte le singole fibre di tal nervo misterioso. Per ora la prego di tener segreta la cosa. Mi obbligherebbe molto ad accennarmi se esiste qualche recentissimo lavoro microscopico intorno al simpatico. 
Non ho nemmeno potuto veder citato nel Jahresbericht: Stannius, Über die fortwährende Metamorphose der Gewebe. Forma esso un libro a parte? L'argomento è interessantissimo.

De-Filippi la saluta e dice che non potè ancora procurarsi la pelle di moufflon.

I spermatozoidi della torpedine sono a guisa di Propfzieher..$^{5}$

Nel contenuto delle cavelle dell'organo elettrico non trovai alcun elemento morfologico.

In dieci giorni spero che sarò a Genova um über die Zitterrochen herzufallen.

Nella lusinga di presto ricevere sue notizie aggradisca i più cordiali saluti dal suo

Obblo. Affo. discepolo e amico Alfonso Corti.

Gentilissima Signora Valentin,

Mille ringraziamenti per le preziosissime righe con cui mi rallegrò ultimamente. Le quali mentre mi dimostrano il ristabilimento di sua salute mi provano altresì che vivo nella di Lei memoria. Giacchè non mi è dato godere della di Lei spiritosa conversazione che in quei brevissimi e pochi giorni di agosto non fece che aumentarne in me il desiderio, sono ben fortunato ch'Ella mi permetta di aggiungerle qualche riga.

Ella m interroga intorno al mio umore (Stimmung). Che debbo dirle? Più avanzo in età e più si concentrano le mie idee sullo studio della natura che è poi la sola pura ed inesausta fonte di soddisfazione. Nella società umana (è un naturalista che parla) il faut tromper ou être trompé. E siccome nè l'una, nè l'altra cosa fa per me, venni alla conclusione che bisogna servirsi della società come ci serviamo del perrucchiere vale a dire pei nostri bisogni veri od immaginarii. Così nel fondo del cuore m'accorgo di diventare pretto misantropo con una veste sociale.

Oltre gli studi ai quali ritorno siccome ai primi amori ho pel capo molti affari di gran momento. Bisogna confessare che l'essere giovane nubile e non insensibile ai pregi od ai vezzi del sesso gentile per eccellenza, è tal cosa troppo spesso incompatibile colla quiete dell'animo. Ammogliarsi adunque, Ella dirà: ma il solo pensiero di ciò aumenta la mia inquietudine, chè «non v'ha rosa senza spina» dice un nostro poeta. Se l'anima

5 Pfropfenzieher, danach folgt im Brief die Zeichnung einer geschlängelten Linie. 
umana fosse accessibile ai nostri sensi e la potessimo notomizzare come sto facendo di alcuni innocenti pesci, vorrei diventare un micrographemonstre, ma quà siamo al bujo, e nè il microscopio, nè la chimica, nè le formole matematiche ci lasciano sperare un fiat lux. Tallerand disse che la parola fu destinata a nascondere il pensiero. Ed io come anatomico sto per dire a dispetto di Lavater e suoi seguaci che la forma materiale dell'uomo che noi appelliamo corpo fu creata per nascondere l'anima. E la scenza ci dimostra che questo stuccio denominato corpo è sempre microscopicamente uguale per tante qualità di anime.

Ma che vado io fantasticando? Sono le due ore dopo mezzanotte e la madre dei pensieri cerca deviarmi dalla retta via dei fatti. Ella scuserà se venni annojandola con simili pazzie esposte in trascurata italiana favella. Si rida adunque un poco del bizzarro anatomico italiano e con ciò avrò ottenuto il mio scopo.

Nella ventura state spero di farle una visita. $\mathrm{E}$ mi prometto gran piacere nel filosofizzare un poco stoicamente su questo mondaccio.

De-Filippi la riverisce tanto.

Mi baci mille volte il Suo Adolfo e la sua Anna e si rammenti qualche volta del Suo

$$
\text { Dev. Serv. }
$$

\section{Alfonso Corti.}

De-Filippi le spedì per occasione per la via di Zurigo la sua lorgnette con altre cose pel di Lei marito.

Soweit Cortis Schreiben. Recht bezeichnend für den Menschen Corti scheinen mir darin die am Schlusse für Frau Valentin beigefügten Zeilen zu sein, verraten sie doch, in welchen Richtungen sich die Gedankengänge des damals 31 jährigen bewegt haben. Da dieser Teil des Briefes allgemeineres Interesse zu erwecken vermag, lasse ich ihn hier noch in möglichst sinngetreuer Übersetzung folgen:

\section{Verehrteste Frau Valentin,}

Tausend Dank für die mir so wertvollen Zeilen, mit denen Sie mich kürzlich erfreuten. Sie zeigen mir einerseits die Wiederherstellung Ihrer Gesundheit an und beweisen mir andererseits, daß ich noch in Ihrer Erinnerung lebe. Sehr beglückt bin ich, daß Sie mir erlauben, einige Zeilen für Sie beizufügen, zumal es mir nicht vergönnt ist, mich Ihrer geist- 
reichen Unterhaltung zu erfreuen; sie hat in jenen allzu kurzen und wenigen Augusttagen nur das Verlangen danach in mir zu steigern vermocht.

Sie fragen mich nach meiner Stimmung. Was soll ich Ihnen sagen? Je älter ich werde, umso mehr konzentrieren sich meine Gedanken auf das Studium der Natur, das schließlich die einzig reine und unerschöpfliche Quelle der Befriedigung darstellt. In der menschlichen Gesellschaft (es ist ein Naturforscher, der spricht) muß man täuschen oder wird getäuscht. Und da mir weder das Eine noch das Andere gefällt, kam ich zu dem Schlusse, daß man sich der Gesellschaft bedienen muß wie wir uns des Friseurs bedienen, das heißt für unsere wahren oder eingebildeten Bedürfnisse. So stelle ich im Grunde meines Herzens fest, daß ich ein reiner Misanthrop - jedoch in geselliger Aufmachung - werde.

Außer den Studien, zu denen ich wie zur ersten Liebe zurückkehre, habe ich viele Dinge von großer Bedeutung im Kopfe. Ich muß bekennen, daß es etwas mit der Seelenruhe allzu oft Unvereinbares ist, jung und unverheiratet und dabei gegen Komplimente und Schmeicheleien des hervorragend liebenswürdigen Geschlechtes nicht unempfindlich zu sein. Also eine Frau nehmen, werden Sie sagen. Aber der bloße Gedanke daran vermehrt meine Unruhe, denn «Keine Rose ohne Dornen» sagt einer unserer Dichter. Wenn die menschliche Seele unseren Sinnen zugänglich wäre und wir sie zergliedern könnten, wie ich es jetzt mit einigen unschuldigen Fischen tue, so möchte ich wohl zu einem Übermikroskopiker werden; aber wir tappen da im Dunkeln und weder das Mikroskop noch die Chemie noch mathematische Formeln lassen uns ein «Es werde Licht» erhoffen. Talleyrand sagte, daß Worte dazu dienen, die Gedanken zu verbergen. Ich als Anatom behaupte trotz Lavater und seinen Anhängern, daß die materielle Form des Menschen, die wir Körper nennen, geschaffen wurde, um die Seele zu verbergen. Die Wissenschaft zeigt uns ja, daß die Hülle, die Körper heißt, bei aller Vielfalt der seelischen Eigenschaften mikroskopisch immer gleich ist.

Aber was phantasiere ich herum? Es ist zwei Uhr nach Mitternacht, und die Mutter der Gedanken sucht mich vom rechten Wege der Tatsachen abzulenken. Sie werden entschuldigen, wenn ich Sie mit derartigen Verrücktheiten in nachlässigem Italienisch langweile. Mögen Sie also ein wenig lachen über den verdrehten italienischen Anatomen, damit habe ich meinen Zweck erreicht.

Im kommenden Sommer hoffe ich Ihnen einen Besuch zu machen. Ich 
verspreche mir ein großes Vergnügen davon, mit ein wenig stoischer Ruhe über diese schlechte Welt zu philosophieren.

De-Filippi läßt sich Ihnen empfehlen. Küssen Sie mir tausend mal Ihren Adolf und Ihre Anna und erinnern Sie sich manchmal an Ihren ergebenen Diener

Alfonso Corti.

De-Filippi schickte Ihnen durch eine Gelegenheit über Zürich Ihre Lorgnette mit anderen Sachen für Ihren Gatten.

Was in Cortis Schreiben an Prof. Valentin über die teils schon ausgeführten, teils nur erst geplanten anatomischen Untersuchungen berichtet wird, interessiert vor allem den Fachmann. Für diesen Teil des Briefes mag deshalb die Wiedergabe des italienischen Textes genügen. Man gewinnt jedenfalls daraus den Eindruck, daß Corti sich mit alter Begeisterung erneut der wissenschaftlichen Arbeit zugewandt hat. Wer würde es für möglich halten, daß er nur wenige Wochen später seine anatomischen Untersuchungen wiederum einstellte, ja sie schließlich gänzlich aufgab? Und doch ist dies nach unseren bisherigen Kenntnissen der Fall. Cortis letztes Schreiben an Prof. Valentin ist vom 8. Januar 1854 datiert, es ist also nur wenige Wochen älter als der hier veröffentlichte Brief. Er bedankt sich darin bei seinem früheren Lehrer, der gerade damals Cortis Wahl zum Mitgliede der Leopoldinisch-Carolinischen Akademie der Naturforscher veranlaßt hatte. Ferner berichtete Corti darin noch über die Ergebnisse einiger mikroskopischer Untersuchungen, aber auch schon über Schwierigkeiten bei der Materialbeschaffung für seine Studien.

In einem am 10. März 1854 verfaßten Dankschreiben an den Präsidenten der eben genannten Akademie, Prof. Nees von Esenbeck in Breslau erwähnte Corti zum ersten Male, daß er durch «eine nicht gefährliche, aber sehr langweilige Krankheit» zur Ruhe genötigt gewesen sei. Dies ist der zeitlich früheste Hinweis auf eine Arthritis deformans, an der Corti nach der Familien-Tradition gelitten haben soll.

Neben dieser Erkrankung müssen aber noch andere Ursachen die Einstellung der anatomischen Arbeit durch Corti mitbedingt haben. Aus brieflichen Mitteilungen des Turiner Zoologen De-Filippi, der zeitweilig mit Corti zusammen gearbeitet hat und ihn sicherlich gut kannte, ist zu ersehen, daß z. B. auch Standesrücksichten - Corti war Marchese dazu beigetragen haben. Wie weit schließlich noch weibliche Einflüsse im Spiele gewesen sind, ist bisher wenig geklärt. Daß Corti trotz seiner 
skeptischen Einstellung gegen das menschliche Wesen das gesellschaftliche Leben nicht gemieden hat, ist aus dem oben stehenden Schreiben an Frau Valentin und aus anderen Äußerungen von ihm bekannt. In diesem Zusammenhang ist jedenfalls noch nötig zu erwähnen, daß Corti seine Bedenken gegen eine eheliche Bindung bald überwunden hat, denn er heiratete im Jahre 1855.

$\mathrm{Ob}$ es je gelingen wird, die tieferen Gründe für die Wesensänderung aufzuklären, durch die Corti der Wissenschaft entfremdet worden ist, bleibt unsicher. Die Familientradition kennt außer der Krankheit keine diesbezüglichen Hinweise. Sollte diese kurze Veröffentlichung bei anderen historisch oder biographisch Interessierten Anteilnahme an Cortis Schicksal erwecken und etwa gar die Beachtung und Auswertung neuer Funde veranlassen, so wäre ihr Zweck in bester Weise erfüllt, denn nur neue Mitteilungen aus Briefen von und über Corti könnten uns helfen, das Leben und die Persönlichkeit dieses so verdienten Forschers genauer kennen zu lernen.

E. Hintzsche.

\section{August von Kekulés dhemische Visionen}

August von Kekulé hat im Jahr 1890 in seiner berühmten Rede anläß. lich des 25. Jahrestages der Aufstellung der Benzolformel dargelegt, daß eine Vision ihn zu dieser Formel geführt habe. Seine Darstellung ist dann in vielen Büchern erwähnt worden und wird natürlich auch in Vorlesungen über Chemie besprochen. Wenn man aber Kekulés eigenen Bericht mit den späteren Wiedergaben vergleicht, dann fällt es auf, daß seine Mitteilungen immer entstellt beschrieben werden. Fast immer werden zwei verschiedene Tatsachen - auf die ich gleich eingehen werde - vermischt, ohne daß man sich einer Verfälschung bewußt wird. Sogar Ernst Mach hat in seinem bekannten Buche «Erkenntnis und Irrtum» (Barth, Leipzig, 5. Aufl. 1926) diesen Fehler begangen und Kekulé's Bericht in ganz unbefriedigender Weise gewissermaßen wegerklärt. Mach schreibt (S. 161):

«Ich bin mit Hallucinationen und Traumphantasien aus eigener Erfahrung wohl vertraut, und mir ist manches optische und musikalische Phantasma vorgekommen, das einer künstlerischen Verwendung wohl fähig wäre. Dagegen kenne ich keinen Fall einer hallucina- 